\title{
Stress in hard metal films
}

\author{
G. C. A. M. Janssen ${ }^{\text {a) }}$ \\ Department of Materials Science and Engineering, Delft University of Technology, Rotterdamseweg 137, \\ 2628 AL Delft, The Netherlands \\ J.-D. Kamminga \\ Netherlands Institute for Metals Research, Rotterdamseweg 137, 2628 AL Delft, The Netherlands
}

(Received 10 May 2004; accepted 17 August 2004)

\begin{abstract}
In the absence of thermal stress, tensile stress in hard metal films is caused by grain boundary shrinkage and compressive stress is caused by ion peening. It is shown that the two contributions are additive. Moreover tensile stress generated at the grain boundaries does not relax by ion bombardment. In polycrystalline hard metal films the grain structure evolves during growth, leading to wider grains higher up in the film. The tensile component of the stress in the film is generated at the grain boundaries and therefore depends on film thickness. The effect of ion bombardment is independent of grain size, therefore compressive stress does not depend on film thickness. As a result in polycrystalline films deposited under a bias voltage a stress gradient exists from tensile at the interface to compressive at the top of the film. (C) 2004 American Institute of Physics.
\end{abstract}

[DOI: 10.1063/1.1807016]

In sputter deposited hard polycrystalline films stresses are regularly observed. Compressive stress is generated by atomic peening: the creation of interstitials or implanted argon in the growing film. ${ }^{1}$ In a previous letter ${ }^{2}$ we have shown that tensile stress in polycrystalline films is inversly proportional to the average grain size. This proportionality rules out vacancy anihilation, pore elimination, and structural relaxation as cause for tensile stress in our polycrystalline films.

In the present letter we discuss the occurrence of stress in sputter deposited chromium films. Chromium films have been deposited at room temperature in a Hauzer HC750 industrial PVD machine on $100 \mathrm{~mm} \mathrm{Si(100)}$ wafers stationary in front of the target, substrate to target distance $25 \mathrm{~cm}$, argon pressure $3 \times 10^{-3}$ mbar. Stresses have been measured by wafer curvature. ${ }^{3}$

In Fig. 1 the stress in $1.1-\mu \mathrm{m}$-thick $\mathrm{Cr}$ films as function of bias voltage is presented. In line with literature stress changes from tensile to compressive in films deposited at increasing bias voltage. ${ }^{4}$ In these $1.1-\mu \mathrm{m}$-thick films the maximum obtained compressive stress was $1 \mathrm{GPa}$. Apparently the stress saturates at $1 \mathrm{GPa}$ for films deposited at bias voltages above $75 \mathrm{~V}$. In Fig. 2 the stress in $\mathrm{Cr}$ films with a thickness ranging from $10 \mathrm{~nm}$ to $3 \mu \mathrm{m}$ is presented. The dots represent films deposited without a bias voltage. The squares represent films deposited under $75 \mathrm{~V}$ bias. The stress in the films deposited without bias is tensile and decreases with increasing thickness. This effect has been observed before. ${ }^{5}$ In a previous letter ${ }^{2}$ we have shown that this tensile stress is generated at the grain boundaries. For the present films the width of the columnar grains is on the order of $30 \mathrm{~nm}$ at a thickness of $100 \mathrm{~nm}$, while on the top of a 1$\mu \mathrm{m}$-thick film the column width is on the order of $60 \mathrm{~nm}$, as observed from TEM micrographs. Since the grain boundary density is lower higher up in the film less stress is generated per unit film thickness higher up in the film. Therefore the average tensile stress decreases with increasing film thickness. The data points in Fig. 2 for deposition without a bias

\footnotetext{
${ }^{a)}$ Electronic mail: g.c.a.m.janssen@tnw.tudelft.nl
}

voltage and a film thickness above $20 \mathrm{~nm}$ were fitted by a power law relation..$^{2}$ The upper curve in Fig. 2 represents

$$
\sigma_{\text {av }}(t)=1.196 \times 10^{7}\left(t^{-0.299}\right)
$$

with $t$ the thickness of the film.

The stress in the films deposited under a bias voltage shows a similar dependence on layer thickness, however shifted to compressive values. Therefore the data points in Fig. 2 obtained with a bias of $75 \mathrm{~V}$ were fitted by

$$
\sigma_{a v}(t)=1.196 \times 10^{7}\left(t^{-0.299}\right)+C .
$$

A good fit is obtained for $C=-1.6 \mathrm{GPa}$. This strongly suggests that stress in films deposited under a bias is an additive result of a thickness-dependent tensile component caused by grain boundary shrinkage and a thicknessindependent compressive component caused by atomic peening. Surprisingly the thickness of the film and thus the average distance from an impinging ion to the nearest grain boundary seems to have no effect on the contribution to the

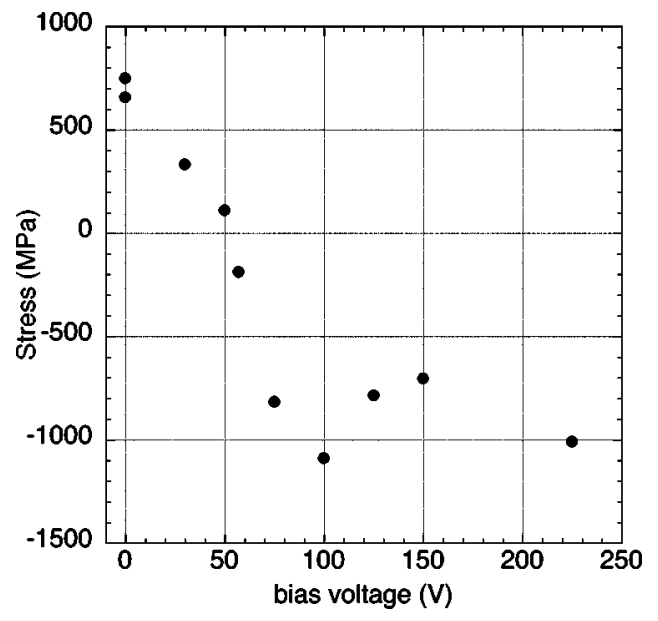

FIG. 1. Stress in 1.1- $\mu$ m-thick Cr films deposited by sputter deposition vs applied bias voltage during deposition. 


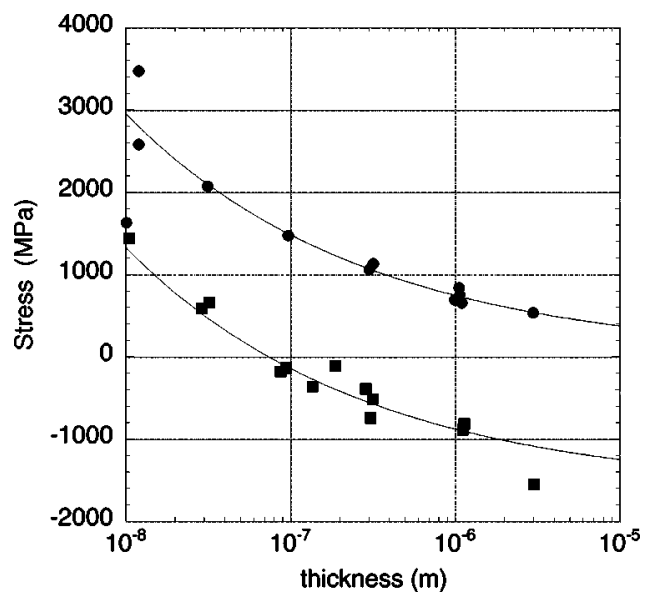

FIG. 2. Stress in chromium films deposited without bias (dots) and under an applied bias of $75 \mathrm{~V}$ (squares). Both series show the same dependence of stress on thickness. The curves are fits based on tensile stress being generated at the grain boundaries (upper curve) and the combined effect of grain boundaries and ion peening (lower curve).

compressive stress. In the films deposited under a bias voltage the stress is tensile for thin films (less than $0.1 \mu \mathrm{m}$ in our case) and compressive for thicker films.

In view of the same dependence on film thickness of the tensile stress component in films deposited with and without bias, the development of the grain boundary density with film thickness should be the same for both conditions. We made TEM cross sections of $1.1-\mu \mathrm{m}$-thick films deposited with and without bias. The films consist of a columnar structure with grains wider at the top than lower in the film. The average grain width is hard to obain from the TEM micrographs but seems comparable for the two cases. According to Eq. (1) the grains should be twice as wide at $1 \mu \mathrm{m}$ as they are at $100 \mathrm{~nm}$. This is in agreement with the general impression from the TEM micrographs. A notable difference in the TEM micrographs, however, is the damage due to ion peening visible in the film deposited under bias. ${ }^{6}$ In both $1.1-\mu \mathrm{m}$-thick films the argon content was measured by wavelength dispersive electron-probe $\mathrm{x}$-ray microanalysis (EPMA) using a JEOL JKA $8900 \mathrm{R}$ micro analyzer. Both films contain 0.01 at. \% Ar. Since the argon concentration has the same low value for both films we ascribe the compressive stress to $\mathrm{Cr}$ interstitials.

A second indication for the similarity of the microstructure follows from XRD measurements performed on 1.1$\mu \mathrm{m}$-thick samples deposited with and without bias. The full width at half maximum of the peaks can be used as a gauge for the microstructure. For all peaks (various reflections were recorded at various specimen tilt angles $\psi$, see the following) the width was the same for the films deposited with and without bias. Point defects do not induce peak broadening. ${ }^{7}$

We will use the distinct features of curvature stress measurements and XRD stress measurements to demonstrate the additivity of stresses caused by atomic peening and grain boundary shrinkage. In Figs. 3 and 4 lattice spacings in $1.1 \mu \mathrm{m}$ films deposited without bias (Fig. 3) and with $75 \mathrm{~V}$ bias (Fig. 4) are presented. Lattice spacings were obtained from 110, 200, 211, and 220 XRD peak positions recorded at various specimen tilt angles $\psi$. The diffractometer was carefully calibrated using a strain free $\mathrm{Al}$ reference powder. The data are represented as $\sin ^{2} \psi$ plots: if the stress state is biaxial, the data should be on a straight line whose slope is

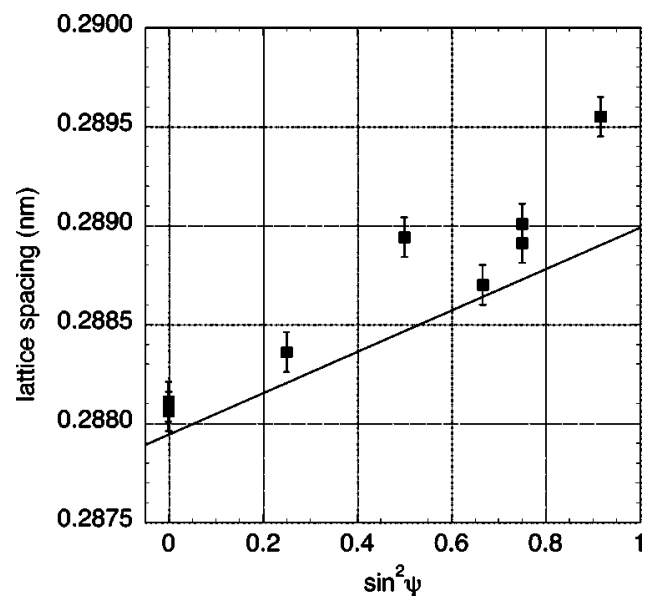

FIG. 3. $\operatorname{Sin}^{2} \psi$ plot of a $1.1-\mu$ m-thick $\mathrm{Cr}$ film under a tensile stress of $840 \mathrm{MPa}$. The lattice spacing in the film is plotted vs the angle $\psi$ between diffraction vector and surface normal. The line represents the lattice spacing calculated from the stress measured by wafer curvature.

proportional to the stress. The lines in Figs. 3 and 4 are lattice spacings calculated from the stress in the films measured by wafer curvature (Fig. 2), assuming a rotationally symmetric biaxial stress state and employing the literature values for the lattice parameter and the elastic constants. In Fig. 3 good agreement between the measured lattice spacings and the calculated spacings is observed. The slope is identical, which shows that the same tensile stress of $840 \mathrm{MPa}$ is obtained from both XRD and wafer curvature, and the measured lattice spacings are only slightly larger than the calculated ones. The good agreement indicates a purely biaxial state of stress. This was expected because the grain boundaries that induce the tensile stress are all oriented along the specimen surface normal. Consequently, they can only exert stress along in-plane directions.

In Fig. 4 the slope of a line through the measurements is again parallel to the calculated line, XRD and wafer curvature yield the same stress. However, the measurements show an appreciable offset to the calculated line. At al angles $\psi$, the lattice spacing is larger than calculated for a biaxial stress state. This effect was recently explained. ${ }^{8,9}$ Due to the threedimensional strain fields around the inclusions caused by

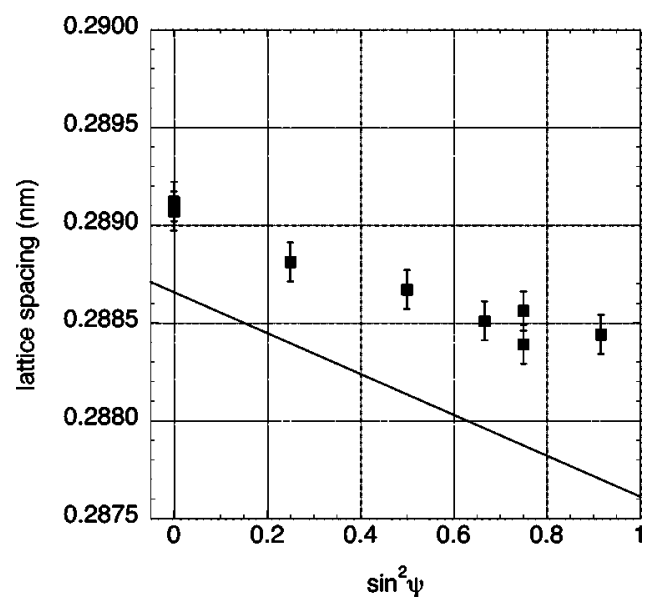

FIG. 4. $\operatorname{Sin}^{2} \psi$ plot of a 1.1- $\mu$ m-thick Cr film under a compressive stress of $890 \mathrm{MPa}$. The lattice spacing in the film is plotted vs the angle $\psi$ between diffraction vector and surface normal. The line represents the lattice spacing calculated from the stress measured by wafer curvature. 


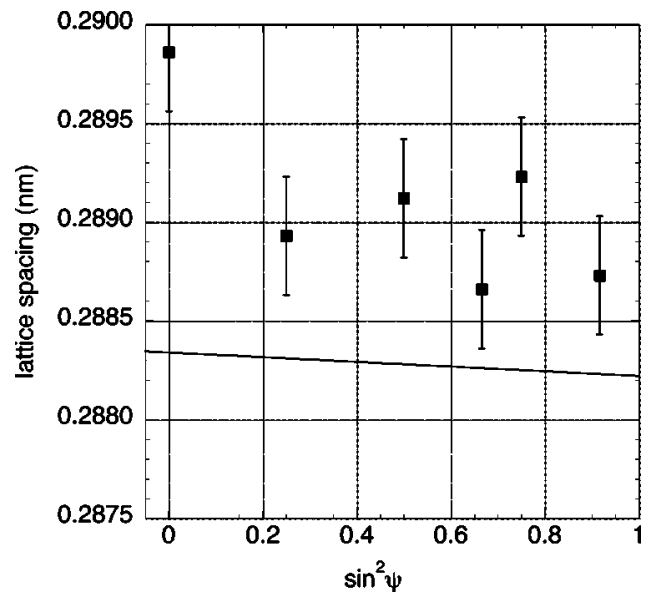

FIG. 5. $\operatorname{Sin}^{2} \psi$ plot of a 90-nm-thick $\mathrm{Cr}$ film under a compressive stress of $100 \mathrm{MPa}$. The lattice spacing in the film is plotted vs the angle $\psi$ between diffracting vector and surface normal. The drawn line represents the lattice spacing calculated from the stress measured by wafer curvature.

atomic peening, an effectively larger strain free lattice parameter is observed with $\mathrm{x}$-ray diffraction, which yields an offset in the $\sin ^{2} \psi$ plot such as the one observed in Fig. 4. The offset in the $\sin ^{2} \psi$ plot can therefore be regarded as proof that atomic peening is effective.

A consequence of the additivity of the stress caused by atomic peening and grain boundary shrinkage is that in a film where the contributions of both mechanisms lead to zero total stress, lattice spacings measured by XRD should be larger than the literature value for the lattice parameter. In Fig. 5 the lattice spacing in a 90-nm-thick $\mathrm{Cr}$ film deposited under $75 \mathrm{~V}$ bias is shown. The stress determined from wafer curvature for this film is only $100 \mathrm{MPa}$ compressive. The calculated lattice spacing is indeed almost constant. It is clear that the measured lattice parameter is dilated with respect to the strain free lattice parameter of chromium at $0.2883 \mathrm{~nm}$ in all directions.
Misra et al. have studied the effect of ion radiation on the stress in previously deposited $\mathrm{Cr}$ films. ${ }^{5}$ In their paper the decrease in tensile stress due to ion bombardment is termed stress relaxation. Even though in their case the ion energy was $300 \mathrm{keV}$ and here the ions have a maximum energy of $75 \mathrm{eV}$ it is possible that also for their experiments tensile stress generated at the grain boundaries and ion peening are unrelated additive effects and the tensile stress is not relaxed but compensated.

In conclusion it is shown that for sputter deposited hard metal films the compressive stress induced by ion peening is additive to the tensile stress generated at grain boundaries. As a consequence the average stress in a film depends on the thickness. A film that is stress free at any thickness will require a deposition strategy with a high ion peening contribution during the initial growth and a lower ion peening contribution later on.

The authors gratefully acknowledge the x-ray diffraction experiments performed by N. van der Pers, the FIB-TEM work by V. Sivel and W.R. Wang, and the EPMA work by W.G. Sloof of our laboratory.

${ }^{1}$ F. M. D’Heurle, Metall. Trans. 1, 725 (1970).

${ }^{2}$ G. C. A. M. Janssen, A. J. Dammers, V. G. M. Sivel, and W. R. Wang, Appl. Phys. Lett. 83, 3287 (2003).

${ }^{3}$ P. A. Flinn, D. S. Gardner, and W. D. Nix, IEEE Trans. Electron Devices ED-34, 689 (1987).

${ }^{4}$ H. Windischmann, Crit. Rev. Solid State Mater. Sci. 17, 547 (1992).

${ }^{5}$ A. Misra, S. Fayeulle, H. Kung, T. E. Mitchell, and M. Nastasi, Nucl. Instrum. Methods Phys. Res. B 148, 211 (1999).

${ }^{6}$ I. Petrov, L. Hultman, U. Helmersson, J.-E. Sundgren, and J. E. Greene, Thin Solid Films 169, 299 (1989).

${ }^{7}$ M. A. Krivoglaz, X-Ray and Neutron Diffraction in Nonideal Crystals (Springer, Berlin, 1996).

${ }^{8}$ J.-D. Kamminga, Th. H. de Keijser, R. Delhez, and E. J. Mittemeijer, J. Appl. Phys. 88, 6332 (2000).

${ }^{9}$ G. Abadias and Y. Y. Tse, J. Appl. Phys. 95, 2414 (2004). 